\title{
NONVANISHING ELEMENTS FOR BRAUER CHARACTERS
}

\author{
SILVIO DOLFI, EMANUELE PACIFICI, AND LUCIA SANUS
}

Dedicated to the memory of Laci Kovács

\begin{abstract}
Let $G$ be a finite group, and $p$ a prime. We say that a $p$-regular element $g$ of $G$ is $p$-nonvanishing if no irreducible $p$-Brauer character of $G$ takes the value 0 on $g$. The main result of this paper shows that if $G$ is solvable and $g \in G$ is a $p$-regular element which is $p$-nonvanishing, then $g$ lies in a normal subgroup of $G$ whose $p$-length and $p^{\prime}$-length are both at most 2 (with possible exceptions for $p \leq 7$ ), the bound being best possible. This result is obtained through the analysis of one particular orbit condition in linear actions of solvable groups on finite vector spaces, and it generalizes (for $p>7$ ) some results in [3].
\end{abstract}

\section{INTRODUCTION AND PRELIMINARIES}

An element $g$ of a finite group $G$ is called a nonvanishing element if $\chi(g) \neq 0$ for every irreducible complex character $\chi \in \operatorname{Irr}(G)$; in other words, $g$ is nonvanishing if the column corresponding to $g$ in the character table of $G$ contains no zero entries. In [7] M. Isaacs, G. Navarro and T. Wolf prove that if $G$ is a finite solvable group and $g \in G$ is a nonvanishing element of odd order, then $g$ lies in the Fitting subgroup $\mathbf{F}(G)$ of $G$. Moreover, in [4] it is shown that if $G$ is any finite group and $g \in G$ is a nonvanishing element of order coprime to 6 , then again $g \in \mathbf{F}(G)$.

In this spirit, given a prime number $p$, we consider elements corresponding to columns with no zero entries in the table of Brauer characters in characteristic $p$. We say that a $p$-regular element $g \in G$ is a $p$-nonvanishing element if $\phi(g) \neq 0$ for every irreducible $p$-Brauer character $\phi \in \operatorname{IBr}_{p}(G)$.

In [3] it is proved that if, for a prime $p>3$, all $p$-regular elements of a finite group $G$ are $p$-nonvanishing (a condition that implies the solvability of $G$ ), then $G$ has $p^{\prime}$-length at most 2. Assuming (as we may, since every $p$-Brauer character of $G$ contains $\mathbf{O}_{p}(G)$ in its kernel) that $\mathbf{O}_{p}(G)=1$, this implies that every $p$-regular element of $G$ lies in $\mathbf{O}_{p^{\prime} p p^{\prime}}(G)$.

The main theorem of the present paper extends this result, in the case $p>7$.

Theorem A. Let $p$ be a prime number greater than 3, let $G$ be a finite solvable group with $\mathbf{O}_{p}(G)=1$, and let $g$ be a $p$-regular element of $G$ that is $p$-nonvanishing. Then $g$ lies in $\mathbf{O}_{p^{\prime} p p^{\prime}}(G)$, unless $p \in\{5,7\}$ and the order of $g$ is divisible by 2 or 3 .

Example 4.1 shows that the above statement is "optimal" in some sense. Our approach to Theorem A consists in studying a related problem about linear actions

Key words and phrases. Finite groups; Brauer characters.

The first and the second author are partially supported by the Italian INdAM-GNSAGA. The third author is partially supported by the Spanish MINECO proyecto MTM2013-40464-P, partly with FEDER funds, and Prometeo2011/030-Generalitat Valenciana. 
on modules. A key tool for our analysis is in fact Theorem 2.6, which deals with solvable groups acting irreducibly on modules over prime fields, and satisfying one particular orbit condition. As shown by Example 4.2, Theorem 2.6 is false in general when $p$ is 5 or 7 and the order of $g$ is divisible by 2 or 3, but we don't know whether exceptions to Theorem A really exist in this case. At any rate, different methods should take over in order to extend Theorem A (possibly for $p \in\{2,3\}$ as well), and this is left as open problem.

Every group considered in this paper is assumed to be a finite group. The only non-standard preliminary concept that needs to be introduced, and that will be central in our discussion, is the following.

Definition. Let $\Omega$ be a finite nonempty set, and let $G$ be a subgroup of $\operatorname{Sym}(\Omega)$. Given an orbit $\mathcal{O}$ for the action of $G$ on $\Omega$, and an element $g$ of $G$, we say that $\mathcal{O}$ is $g$-deranged if $g$ does not fix any element in $\mathcal{O}$.

Observe that a regular orbit is clearly $g$-deranged for every nontrivial $g \in G$.

\section{Deranged orbits on the POWER Set}

Definition 1.1. Let $\Omega$ be a finite nonempty set. Given a positive integer $t$, we define $\mathcal{P}_{t}(\Omega)$ to be the set of ordered $(t+1)$-tuples $\left(\Omega_{1}, \Omega_{2}, \ldots, \Omega_{t+1}\right)$, where the $\Omega_{j}$ are (possibly empty) subsets of $\Omega$ such that $\Omega_{j} \cap \Omega_{l}=\emptyset$ whenever $j \neq l$, and $\bigcup_{j=1}^{t+1} \Omega_{j}=\Omega$. We shall write $\mathcal{P}(\Omega)$ rather than $\mathcal{P}_{1}(\Omega)$. (Note that $\mathcal{P}(\Omega)$ can be identified with the power set of $\Omega$.)

Observe that, if $G$ is a subgroup of $\operatorname{Sym}(\Omega)$, then $G$ also embeds into $\operatorname{Sym}\left(\mathcal{P}_{t}(\Omega)\right)$ in a natural way (under the convention that the empty set is fixed by every element of $G$ ). Moreover, there is an obvious bijection between $\mathcal{P}_{t}(\Omega)$ and the subset of $\mathcal{P}_{t+1}(\Omega)$ consisting of the elements $\left(\Omega_{1}, \Omega_{2}, \ldots, \Omega_{t+2}\right)$ such that $\Omega_{t+2}=\emptyset$; this subset is clearly $G$-invariant, and the action of $G$ on it is equivalent to that on $\mathcal{P}_{t}(\Omega)$. For $g \in G$, we shall freely use the obvious fact that, whenever there exists a $g$-deranged orbit for the action of $G$ on $\operatorname{Sym}\left(\mathcal{P}_{t}(\Omega)\right)$, the same happens for the action of $G$ on $\operatorname{Sym}\left(\mathcal{P}_{t+1}(\Omega)\right)$.

Notation 1.2. Let $\Omega$ be a finite set with $|\Omega|>1$, let $G$ be a transitive subgroup of $\operatorname{Sym}(\Omega)$, and $\Delta$ a minimal nontrivial block for the action of $G$ on $\Omega$ (i.e., $|\Delta|>1$, but we allow $\Delta=\Omega$ ). Denoting by $G_{\Delta}$ the stabilizer of $\Delta$ in $G$, and by $L$ the pointwise stabilizer of $\Delta$ in $G$, set $H=G_{\Delta} / L$. In this situation, $H$ can be identified with a primitive subgroup of $\operatorname{Sym}(\Delta)$. Also, let $\Sigma$ be a right transversal for $G_{\Delta}$ in $G$; in view of Remarks 2.1 and 2.2 of [3], $G$ can be identified with a subgroup of $H \imath K$, where $K \leq \operatorname{Sym}(\Sigma)$ is a homomorphic image of $G$ acting transitively on $\Sigma$. Furthermore, the group $H \nmid K$ (thus $G$, as well) acts naturally on the cartesian product $\Delta \times \Sigma$, and the $G$-sets $\Omega$ and $\Delta \times \Sigma$ are equivalent. If $|\Sigma|=s$, then we identify $\Sigma$ with $\{1,2, \ldots, s\} \subseteq \mathbb{N}$. Finally, we write $B$ for the base group of $H \prec K$.

Lemma 1.3. Assuming the setting of Notation 1.2, consider an element $g=$ $\left(h_{1}, h_{2}, \ldots, h_{s}\right) k$ of $G$. Then, for every positive integer $t$, the following conclusions hold.

(a) Let $g$ be in $G \cap B$ (i.e., let $k=1$ ). If, for a given $i \in\{1, \ldots, s\}$, there exists an $h_{i}$-deranged orbit for the action of $H$ on $\mathcal{P}_{t}(\Delta)$, then there exists a $g$-deranged orbit for the action of $G$ on $\mathcal{P}_{t}(\Omega)$. 
(b) If there exists a $k$-deranged orbit for the action of $K$ on $\mathcal{P}_{t}(\Sigma)$, then there exists a g-deranged orbit for the action of $G$ on $\mathcal{P}_{t}(\Omega)$.

Proof. We start by proving part (a) of the statement. Let $\left(\Delta_{1}, \Delta_{2}, \ldots, \Delta_{t+1}\right)$ be an element lying in an $h_{i}$-deranged orbit for the action of $H$ on $\mathcal{P}_{t}(\Delta)$. We define an ordered $(t+1)$-tuple of subsets of $\Delta \times \Sigma$, setting

$$
\Omega_{j}=\left\{(\delta, i) \mid \delta \in \Delta_{j}, i \in\{1, \ldots, s\}\right\}
$$

for every $j \in\{1, \ldots, t+1\}$. We claim that $g$ does not fix any element in the $G$-orbit of $\left(\Omega_{1}, \Omega_{2}, \ldots, \Omega_{t+1}\right)$.

In fact, for a proof by contradiction, assume that there exists $x \in G$ such that $g$ fixes $\left(\Omega_{1}, \Omega_{2}, \ldots, \Omega_{t+1}\right) \cdot x$. Write $x=\left(l_{1}, \ldots, l_{s}\right) z$ for suitable $l_{i} \in H$ and $z \in K$, whence $x g=\left(l_{1} h_{1 \cdot z}, l_{2} h_{2 \cdot z}, \ldots, l_{s} h_{s \cdot z}\right) z$. For $j \in\{1, \ldots, t+1\}$ and $\delta \in \Delta_{j}$, take $r \in\{1, \ldots, s\}$ and $\epsilon \in \Delta$ such that

$$
\left(\delta, i \cdot z^{-1}\right) \cdot x g=(\epsilon, r) \cdot x
$$

holds. Note that $r=i \cdot z^{-1}$, and observe also that $\epsilon$ lies in $\Delta_{j}$, because $g^{x^{-1}}$ fixes $\left(\Omega_{1}, \Omega_{2}, \ldots, \Omega_{t+1}\right)$. Moreover, $\delta \cdot l_{i \cdot z^{-1}} h_{i}=\epsilon \cdot l_{i \cdot z^{-1}}$ holds; thus, setting $u=l_{i \cdot z^{-1}} \in$ $H$, we have that $\delta \cdot h_{i}^{u^{-1}}$ lies in $\Delta_{j}$. Since this holds for every $\delta \in \Delta_{j}$, we conclude that $h_{i}^{u^{-1}}$ fixes $\Delta_{j}$ and, as this happens for every $j \in\{1, \ldots, t+1\}$, we get that $h_{i}$ fixes $\left(\Delta_{1}, \Delta_{2}, \ldots, \Delta_{t+1}\right) \cdot u$, contradicting the fact that $\left(\Delta_{1}, \Delta_{2}, \ldots, \Delta_{t+1}\right)$ lies in an $h_{i}$-deranged orbit for the action of $H$ on $\mathcal{P}_{t}(\Delta)$.

We move now to part (b). Let $\left(\Sigma_{1}, \Sigma_{2}, \ldots, \Sigma_{t+1}\right)$ be an element of $\mathcal{P}_{t}(\Sigma)$ lying in a $k$-deranged orbit. Setting

$$
\Omega_{j}=\left\{(\delta, i) \mid \delta \in \Delta, i \in \Sigma_{j}\right\}
$$

for $j \in\{1, \ldots, t+1\}$, we claim that $\left(\Omega_{1}, \Omega_{2}, \ldots, \Omega_{t+1}\right)$ lies in a $g$-deranged orbit for the action of $G$ on $\mathcal{P}_{t}(\Omega)$. In fact, as above, let $x \in G$ be such that $g$ fixes $\left(\Omega_{1}, \Omega_{2}, \ldots, \Omega_{t+1}\right) \cdot x$. Write $x=\left(l_{1}, \ldots, l_{s}\right) z$, with $l_{i} \in H$ and $z \in K$, whence $g^{x^{-1}}=\left(y_{1}, y_{2}, \ldots, y_{s}\right) k^{z^{-1}}$ for suitable $y_{i} \in H$. For $\delta \in \Delta$ and $i \in \Sigma_{j}$, we get $(\delta, i) \cdot g^{x^{-1}}=\left(\delta \cdot y_{i}, i \cdot k^{z^{-1}}\right)$ : as $g^{x^{-1}}$ stabilizes $\Omega_{j}$, we have $i \cdot k^{z^{-1}} \in \Sigma_{j}$ and therefore $k^{z^{-1}}$ stabilizes $\Sigma_{j}$. As this happens for every $j \in\{1, \ldots, t+1\}$, this contradicts our choice of $\left(\Sigma_{1}, \Sigma_{2}, \ldots, \Sigma_{t+1}\right)$ as an element of $\mathcal{P}_{t}(\Sigma)$ lying in a $k$-deranged orbit, and the proof is complete.

Theorem 1.4. Let $\Omega$ be a finite nonempty set, $G$ a transitive solvable subgroup of $\operatorname{Sym}(\Omega)$, and $g$ a nontrivial element of $G$. Then the following conclusions hold.

(a) There exists a $G$-regular orbit in $\mathcal{P}_{4}(\Omega)$.

(b) There exists a g-deranged orbit in $\mathcal{P}_{3}(\Omega)$.

(c) If there does not exist any $g$-deranged orbit in $\mathcal{P}_{2}(\Omega)$, then $g$ lies in $\mathbf{O}_{\{2,3\}}(G)$.

(d) If there does not exist any $g$-deranged orbit in $\mathcal{P}(\Omega)$, then $g$ is a $\{2,3\}$-element of $G$ lying either in $\mathbf{O}_{\{2,3,5\}}(G)$ or in $\mathbf{O}_{\{2,3,7\}}(G)$.

Proof. Conclusion (a) is Theorem 1.2 of [10]. We will start by proving parts (b), (c) and (d) under the additional assumption that $G$ is a primitive subgroup of $\operatorname{Sym}(\Omega)$.

In this case, Lemma 1 of [2] guarantees the existence of a $G$-regular orbit on $\mathcal{P}_{2}(\Omega)$ unless $|\Omega|=4$ and $G$ is $\operatorname{Sym}(4)$, so (c) is proved; moreover, the $G$-orbit on $\mathcal{P}_{3}(\Omega)$ containing $(\{1\},\{2\},\{3\},\{4\})$ is regular, thus (b) holds too. As regards the action of $G$ on $\mathcal{P}(\Omega)$, we can clearly assume that $G$ does not have any regular orbit 
on $\mathcal{P}(\Omega)$, so we are in a position to apply Theorem 5.6 of [8]. As we can also assume that $G$ is not a $\{2,3\}$-group, we have to consider the following cases.

(1) $\Omega=\{1,2,3,4,5\}$ and $G \simeq D_{10}$. Then the $G$-orbit of $(\{1,2\},\{3,4,5\})$ in $\mathcal{P}(\Omega)$ is $g$-deranged for any $g \in G$ which is not an involution.

(2) $\Omega=\{1,2,3,4,5\}$ and $G$ is the Frobenius group of order 20 . Then the $G$-orbit on $\mathcal{P}(\Omega)$ containing $(\{1,2\},\{3,4,5\})$ is $g$-deranged for any $g \in G$ that is not an involution.

(3) $\Omega=\{1,2,3,4,5,6,7\}$ and $G$ is the Frobenius group of order 42 . Then the $G$-orbit on $\mathcal{P}(\Omega)$ containing $(\{1,2\},\{3,4,5,6,7\})$ is $g$-deranged for any $g \in G$ that is not an involution.

(4) $\Omega=\{1,2,3,4,5,6,7,8\}$ and $G=A \Gamma\left(2^{3}\right)$ is the affine semilinear group on the field $\operatorname{GF}\left(2^{3}\right)$ (see [8, page 38]). Now, the stabilizer in $G$ of $(\{1\},\{2,3,4,5,6,7,8\})$ has odd order and the stabilizer of $(\{1,2\},\{3,4,5,6,7,8\})$ has order coprime to 7 , so there exists a $g$-deranged orbit in $\mathcal{P}(\Omega)$ for any $g \in G$ which is not of order 3 .

The analysis of the primitive case is complete, and we will henceforth assume that the action of $G$ on $\Omega$ is imprimitive. In what follows we will make use of Notation 1.2. Observe that, taking into account part (a) of Lemma 1.3, conclusions (b), (c) and (d) are easily extended from the primitive to the imprimitive case whenever $g$ lies in $G \cap B$.

Consider first the case $\Delta=\left\{\delta_{1}, \ldots, \delta_{d}\right\}$ with $d \geq 4$. Set $\Delta_{0}=\emptyset$ and, for $i \in\{1, \ldots, 4\}$, set $\Delta_{i}=\left\{\delta_{1}, \ldots, \delta_{i}\right\}$. Now, by part (a) we can choose an element $\left(\Sigma_{0}, \ldots, \Sigma_{4}\right)$ lying in a regular $K$-orbit for the action of $K$ on $\mathcal{P}_{4}(\Sigma)$, and we can define

$$
\Omega_{1}=\bigcup_{j=0}^{4}\left\{(\delta, i) \mid \delta \in \Delta_{j}, i \in \Sigma_{j}\right\} .
$$

Writing $g=\left(h_{1}, \ldots, h_{s}\right) k$, assume that the $G$-orbit of $\left(\Omega_{1}, \Omega \backslash \Omega_{1}\right)$ in $\mathcal{P}(\Omega)$ is not $g$-deranged, i.e., there exists $x=\left(l_{1}, \ldots, l_{s}\right) z \in G$ such that $g$ fixes $\left(\Omega_{1}, \Omega \backslash \Omega_{1}\right) \cdot x$. Then $g^{x^{-1}}$, which we write $\left(y_{1}, y_{2}, \ldots, y_{s}\right) k^{z^{-1}}$ for suitable $y_{i} \in H$, fixes $\left(\Omega_{1}, \Omega \backslash \Omega_{1}\right)$, and therefore $k^{z^{-1}}$ fixes $\left(\Omega_{1}, \Omega \backslash \Omega_{1}\right) \cdot\left(y_{1}, y_{2}, \ldots, y_{s}\right)$. It is not hard to check that this forces $k^{z^{-1}}$ to fix $\left(\Sigma_{0}, \ldots, \Sigma_{4}\right)$, so that $k$ is trivial and $g$ lies in $G \cap B$. As observed in the paragraph above, we are done in this case.

It remains to treat the situation when $|\Delta| \leq 3$, which implies that $H$ is a $\{2,3\}$ group. Write $g=\left(h_{1}, \ldots, h_{s}\right) k$. If $k=1$, then again we are done; if $k \neq 1$ then, by induction on the cardinality of the permuted set, conclusions (b), (c), (d) of the statement hold with respect to the action of $K$ on $\Sigma$ and to the element $k$, and an application of part (b) of Lemma 1.3 concludes the proof.

\section{Deranged orbits in LINEAR ACTIONS}

The structure of primitive solvable groups of linear transformations is quite well understood. In the following proposition we collect some well-known facts (see for instance [11, Theorem 2.2 and Lemma 2.4], [8, Lemma 0.5 and Theorem 1.9]) and, after that, we will be ready to describe some features of these groups that will be relevant for our purposes. 
Proposition 2.1. Let $G$ be a solvable group, and $V$ a faithful primitive $G$-module over a finite field. Then there exist subgroups $Z \leq U \leq F \leq A$, and $E$, all normal in $G$, with the following properties.

(a) $U$ is cyclic, and $E$ is a product of subgroups $E_{i} \triangleleft G$ of pairwise coprime orders, such that, for every $i, E_{i}$ is cyclic of prime order $p_{i}$ or an extraspecial $p_{i}$-group (of exponent $p_{i}$ if $p_{i} \neq 2$, and of order $p_{i}^{2 n_{i}+1}$ for a suitable integer $n_{i}$ ). Also, $F=E U$ is a central product, $Z=E \cap U=\mathbf{Z}(E)$ and $\mathbf{C}_{G}(F) \leq F$.

(b) $F / U \simeq E / Z$ is a direct sum of completely reducible $G / F$-modules.

(c) $A=\mathbf{C}_{G}(U)$, so that $G / A$ embeds into the abelian group $\operatorname{Aut}(U)$.

(d) $A / F$ acts faithfully on $E / Z$, and $A / \mathbf{C}_{A}\left(E_{i} / \mathbf{Z}\left(E_{i}\right)\right)$ embeds into the symplectic group $\operatorname{Sp}\left(2 n_{i}, p_{i}\right)$.

(e) If $W$ an irreducible submodule of $V_{U}$, then $U$ acts fixed-point freely on $W$ (hence $|U|$ divides $|W|-1)$ and $|G: A|$ is a divisor of $\operatorname{dim}(W)$.

(f) Setting $e=\sqrt{|E: Z|}$, we have $|V|=|W|^{e b}$ for some integer $b$.

(g) Let $g$ be an element of prime order $r$ in $G$. Then $\left|\mathbf{C}_{V}(g)\right| \leq|V|^{1 / 2}$, unless $g$ lies in $A \backslash F$ and either $r=2$ or $r$ divides $|E|$. In any case, we have $\left|\mathbf{C}_{V}(g)\right| \leq|V|^{3 / 4}$.

Theorem 2.2. Let $G$ be a solvable group, $p$ a prime number greater than 3 , and $V$ a faithful primitive $G$-module over a prime field. If there exists a nontrivial $p$ regular element $g$ of $G$ such that there do not exist $g$-deranged orbits in $V$, then the p-length of $G$ is at most 1.

Proof. Let $g$ be an element of $G$ as in the statement. Since $G$ acts faithfully and primitively on $V$, we can use Proposition 2.1 and the notation introduced therein. Observe that, since there are not $g$-deranged orbits for the action of $G$ on $V$, by Theorem 4.1 of [11] we have $e \in\{2,3,4,8,9,16\}$. Hence, $E$ is an extraspecial $r$ group with $r \in\{2,3\}$ (so $r \neq p$ ). Write $A=\mathbf{C}_{G}(U)$, and notice that $U$ contains $\mathbf{O}_{p}(A)$, which is therefore central in $A$. If $A / F$ is a $p^{\prime}$-group, then clearly $\mathbf{O}_{p^{\prime} p}(A)=$ $A$ and, as $G / A$ is abelian, $G=\mathbf{O}_{p^{\prime} p p^{\prime}}(G)$. In view of that, in what follows we will assume that $p$ divides $|A / F|$ (thus, in particular, $A / F$ is not a $\{2,3\}$-group).

If $e \in\{2,3\}$, then $A / F$ embeds into $\mathrm{GL}(2,2)$ or $\mathrm{GL}(2,3)$ respectively. In any case, $A / F$ is a $\{2,3\}$-group against our assumption.

Thus, we have $e \in\{4,8,9,16\}$; in the following discussion we will refer to the analysis carried out in Lemma 3.2 and Lemma 3.3 of [1], and our aim will be to show at first that $A=\mathbf{O}_{p^{\prime} p p^{\prime}}(A)$.

If $e=4$, then $A / F$ embeds in $\operatorname{Sp}(4,2) \simeq \operatorname{Sym}(6)$, which is a $\{2,3,5\}$-group; moreover, either $A / F \leq \mathrm{GL}(2,2)$ 乙 $\operatorname{Sym}(2)$, or $A / F \leq \Gamma\left(2^{4}\right)$, the semilinear group on the field with $2^{4}$ elements. As $\operatorname{Sym}(6)$ has no elements of order 15 , we see that $A / F$ is a $\{2,5\}$-group of order at most 20 and that if 5 divides the order of $A / F$, then $A / F$ has cyclic Sylow 2-subgroups. In this case $A / F$ has a normal Sylow $p$-subgroup. Hence, $A / F=\mathbf{O}_{p p^{\prime}}(A / F)$ and it follows that $A=\mathbf{O}_{p^{\prime} p p^{\prime}}(A)$.

If $e=8$, then $A / F$ embeds in $\operatorname{Sp}(6,2)$, and we get either $A / F \leq \operatorname{GL}(2,2) \times \Gamma\left(2^{4}\right)$ (with the projection on the second factor of order at most 20 ) or $A / F \leq \mathrm{GL}(2,2) \times$ $\Gamma\left(2^{3}\right)$, or $A / F \leq \Gamma\left(2^{6}\right)$ with $|A / F| \leq 42$. Thus $p=5$ or $p=7$, and in any case $A / F$ has a normal Sylow $p$-subgroup. Again it follows $A / F=\mathbf{O}_{p p^{\prime}}(A / F)$ and thus $A=\mathbf{O}_{p^{\prime} p p^{\prime}}(A)$.

If $e=9$, then $A / F \leq \mathrm{Sp}(4,3)$ and we have to consider two possibilities. 
(i) $A / F \leq 2 . \operatorname{Sym}(6)$. Then, looking at the solvable subgroups of $2 . \operatorname{Sym}(6)$, we deduce that $A / F$ has order at most 40 , so that $p=5$ and $A / F$ has a normal Sylow $p$-subgroup. Hence, $A / F=\mathbf{O}_{p p^{\prime}}(A / F)$ and $A=\mathbf{O}_{p^{\prime} p p^{\prime}}(A)$.

(ii) $A / F \leq\left(D_{8}\right.$ Y $\left.Q_{8}\right)$. Alt $(5)$. Then, looking at the solvable subgroups of $\left(D_{8} Y\right.$ $\left.Q_{8}\right)$.Alt (5), we see that $A / F \leq\left(D_{8} Y Q_{8}\right)$. $D_{10}$, whence $|A / F| \leq 320$ and $p=5$. Moreover, setting $L / F=\mathbf{O}_{2}(A / F)$, we get that $L$ has a normal $p$-complement because $\mathbf{O}_{p}(F)$ lies in $\mathbf{Z}(A)$, and $A / L$ has a normal Sylow $p$-subgroup. It follows that $A=\mathbf{O}_{p^{\prime} p p^{\prime}}(A)$.

Finally we consider the case $e=16$, so that $A / F$ embeds in $\operatorname{Sp}(8,2)$, and we have the following possibilities.

(i) $A / F \leq L$, where $L$ is a group among $(\operatorname{GL}(2,2) \imath \operatorname{Sym}(2)) \times \Gamma\left(2^{4}\right), \Gamma\left(2^{4}\right) \times \Gamma\left(2^{4}\right)$, $\Gamma\left(2^{4}\right) \imath \operatorname{Sym}(2)$ and $\Gamma\left(2^{4}\right) .2$. It can be checked that $p=5$ and $A / F$ has a normal Sylow 5-subgroup.

(ii) $A / F \leq L$, where $L$ is a group among $(\operatorname{GL}(2,2)$ ? $\operatorname{Sym}(2)) \times \Gamma\left(2^{3}\right), \operatorname{GL}(2,2) \times$ $\Gamma\left(2^{3}\right) \times \Gamma\left(2^{3}\right), \mathrm{GL}(2,2) \times\left(\Gamma\left(2^{3}\right) \cdot 2\right)$ or $\mathrm{GL}(2,2) \times \Gamma\left(2^{6}\right)$. In this case, $p=7$ and $A / F$ has a normal Sylow 7 -subgroup.

(iii) $A / F \leq \Gamma\left(2^{3}\right) \times \Gamma\left(2^{4}\right)$. In this case $p \in\{5,7\}$, and $A / F$ has a normal Sylow p-subgroup.

(iv) $A / F \leq \Gamma\left(2^{8}\right)$. Here $p \in\{5,51\}$, and $A / F$ has a normal Sylow $p$-subgroup.

(Note that, in any case, $|A / F| \leq 7200$.)

Our conclusion so far is that $A=\mathbf{O}_{p^{\prime} p p^{\prime}}(A)$ and, in order to conclude the proof, it will be enough to show that $G / A$ is a $p^{\prime}$-group whenever there exists $g \in G \backslash\{1\}$ such that there are no $g$-deranged orbits in $V$. Aiming at a contradiction, we will assume that $G$ has $p$-length bigger that 1 ; so $p$ divides $|G / A|$ and, in particular, $|G / A| \geq 5$.

Observe that the lack of $g$-deranged orbits in $G$ implies

$$
\bigcup_{x \in G} \mathbf{C}_{V}\left(g^{x}\right)=V
$$

As there are clearly no deranged orbits in $V$ for any power of $g$, we can assume that $g$ is an element of prime order $r$. Hence we get $\left|\mathbf{C}_{V}(g)\right| \leq|V|^{\alpha}$, where $\alpha$ takes the value $1 / 2$ or $3 / 4$ according to part (g) of Proposition 2.1, and thus

$$
|V| \leq\left|G: \mathbf{N}_{G}(\langle g\rangle)\right| \cdot|V|^{\alpha} .
$$

Since the number of $G$-conjugates of $\langle g\rangle$ is easily seen to be at most $|A|$, we get

$$
|V|^{1-\alpha} \leq|A / F| \cdot|U| \cdot|E / Z| \text {. }
$$

However, recall that $U$ lies in $\mathbf{Z}(A)$; therefore, if $g$ is in $A$, the number of $G$ conjugates of $\langle g\rangle$ is also bounded by $|G / A| \cdot|A / F| \cdot|E / Z|$, and we have

$$
|V|^{1-\alpha} \leq|G / A| \cdot|A / F| \cdot|E / Z| \text {. }
$$

In what follows, using the notation of Proposition 2.1, we set $|W|=q^{a}$ where $q$ is a suitable prime; thus part (f) of that proposition yields $|V|=q^{e a b}$, whereas part (e) yields $|U|<q^{a}$ and $a=k|G / A|$ for some $k \in \mathbb{Z}$.

Let us consider first the case $e=4$. looking at the discussion in the first part of this proof, we see that $|A / F| \leq 20$ and $p=5$, so $a$ is a multiple of 5 . Note also that $|U|$ is an even divisor of $q^{a}-1$, so that $q$ is an odd prime. If $g \notin A$, then inequality (2) yields

$$
q^{2 a b}<20 \cdot q^{a} \cdot 2^{4}
$$


whence $q^{a}<2^{6} \cdot 5$. This forces $q=3$ and $a=|G / A|=5$. Now 5 is a divisor of the order of $g$, contradicting the fact that $g$ is a $p^{\prime}$-element. Therefore $g$ lies in $A$ and we can use inequality (3); if $g$ is not an involution in $A \backslash F$, then we obtain $3^{10} \leq 5 \cdot 20 \cdot 2^{4}$, which is false. We conclude that $g$ is an involution in $A \backslash F$, and at any rate inequality (3) yields

$$
q^{k|G / A| b} \leq|G / A| \cdot 20 \cdot 2^{4} .
$$

It is easy to see that this implies $q=3, a=|G / A|=5$ and $b=1$. Moreover, $|U|$ divides $3^{5}-1=2 \cdot 11^{2}$. By inequality (1), $|V|=3^{20} \leq\left|G: \mathbf{N}_{G}(\langle g\rangle)\right| \cdot|V|^{3 / 4}$; so $\left|G: \mathbf{N}_{G}(\langle g\rangle)\right| \geq 3^{5}$. Moreover, $|A / F|$ is divisible by 5 (as otherwise $G$ has $p$-length $1)$, so $A / F$ can be identified with a subgroup of order 10 or 20 of $\Gamma\left(2^{4}\right)$, acting on $F / U$. It follows that $A / U$ contains 20 involutions and hence $A$ contains 40 involutions (besides the involution of $U$, that is central in $A$ and certainly not a conjugate of $g$ ), against $\left|G: \mathbf{N}_{G}(\langle g\rangle)\right| \geq 3^{5}$.

Assume next $e=8$; in this case, we know that $|A / F| \leq 2 \cdot 3^{2} \cdot 7$. Inequality (2) yields $q^{a}<2^{7} \cdot 3^{2} \cdot 7$ (with $a \geq 5$ ), thus forcing $q \in\{3,5\}$ and $a=|G / A| \in\{5,7\}$. In any case we get $p=|G / A|$, therefore, $g$ being a $p^{\prime}$-element, we get $g \in A$. Using now inequality $(3)$, we obtain $3^{10} \leq 2^{7} \cdot 3^{2} \cdot 7^{2}$, which is false. Therefore $e$ cannot be 8 under our assumptions.

As for the case $e=9$, we have $|A / F| \leq 320$ and $p=5$, thus $a$ is a multiple of 5. Assume first $g \notin A$; then we can use inequality (2) with $\alpha=1 / 2$, obtaining $q^{9 a / 2}<320 \cdot q^{a} \cdot 3^{4}$. Hence, we have $q^{35 / 2}<320 \cdot 3^{4}$, which is false for every prime $q$. On the other hand, assume $g \in A$. Now we can use inequality (3), which yields

$$
q^{9 k|G / A| b / 4} \leq|G / A| \cdot 320 \cdot 3^{4} .
$$

Even setting $b=1$, this forces $q=2$ and $a=|G / A|=5$. But $|U|$ is a multiple of 3 in this case, and $|U|$ should divide $2^{5}-1=31$, a clear contradiction. Thus also the value 9 for $e$ is impossible.

Finally, consider the case $e=16$, thus $|A / F| \leq 7200$. Inequality (2) yields

$$
q^{4 a b}<7200 \cdot q^{a} \cdot 2^{8},
$$

that is $q^{3 a}<7200 \cdot 2^{8}$. This implies $q=2$, a contradiction.

The proof is now complete.

The conclusion of the above theorem also holds for solvable groups inducing few orbits in primitive linear actions.

Lemma 2.3. Let $G$ be a solvable group, $p$ a prime number greater than 3 , and $V$ a faithful primitive $G$-module over a prime field. If the elements of $V$ are partitioned in at most three orbits under the action of $G$, then the p-length of $G$ is at most 1.

Proof. By [8, Theorem 6.8] and [5, Theorem 1.1] (which deal with the case when the number of $G$-orbits on $V \backslash\{0\}$ is 1 or 2 , respectively), it is easily checked that either $G$ is a group of semilinear maps on $V$, or $G$ belongs to a list of groups whose orders are not divisible by $p^{2}$ whenever $p$ is a prime greater than 3 . Therefore, the desired conclusion follows.

In order to investigate imprimitive linear actions from the relevant point of view, in the spirit of Notation 1.2 we establish the following setting. 
Notation 2.4. Let $G$ be a group, and $V$ an irreducible $G$-module. Choosing a subgroup $T$ of $G$ and a primitive submodule $W$ of $V_{T}$ such that $V=W^{G}$ (possibly $T=G)$, we set $H=T / \mathbf{C}_{T}(W)$. Also, we denote by $\Sigma$ a right transversal for $T$ in $G$ and, if $s=|\Sigma|$, we identify $\Sigma$ with $\{1,2, \ldots, s\} \subseteq \mathbb{N}$. As already mentioned in Notation 1.2, $G$ can be identified with a subgroup of $H \imath K$, where $K$ is a transitive subgroup of $\operatorname{Sym}(\Sigma)$; moreover, $H \imath K$ (thus $G$ ) acts naturally on the direct sum $W^{\oplus s}$ of $s$ copies of $W$, and the $G$-modules $V$ and $W^{\oplus s}$ are isomorphic (see Remark 2.3 of [3]). Finally, we denote by $B$ the base group of $H$ ? $K$.

Next, we gather the analogue of Lemma 1.3 in the context of linear actions.

Lemma 2.5. Assuming the setting of Notation 2.4, consider an element $g=$ $\left(h_{1}, h_{2}, \ldots, h_{s}\right) k$ of $G$. Then the following conclusions hold.

(a) Let $g$ be in $G \cap B$ (i.e., let $k=1$ ). If, for a given $i \in\{1, \ldots, s\}$, there exists an $h_{i}$-deranged orbit for the action of $H$ on $W$, then there exists a $g$-deranged orbit for the action of $G$ on $V$.

(b) Let $t$ be a positive integer. If there exists a $k$-deranged orbit for the action of $K$ on $\mathcal{P}_{t}(\Sigma)$, and $W$ is partitioned in at least $t+1$ orbits under the action of $H$, then there exists a $g$-deranged orbit for the action of $G$ on $V$.

Proof. In order to prove (a), let us choose an element $w$ of $W$ lying in an $h_{i}$-deranged orbit for the action of $H$, and consider the element $v \in W^{\oplus s}$ whose $i$ th component is $w$ for every $i \in\{1, \ldots, s\}$. We claim that $v$ lies in a $g$-deranged orbit for the action of $G$. In fact, assume that there exists $x \in G$ such that $v^{x g}=v^{x}$. Write $x=$ $\left(l_{1}, \ldots, l_{s}\right) z$ for suitable $l_{i} \in H$ and $z \in K$, whence $x g=\left(l_{1} h_{1 \cdot z}, l_{2} h_{2 \cdot z}, \ldots, l_{s} h_{s \cdot z}\right) z$. We have

$$
v^{x g}=\left(w^{l_{1} h_{1 \cdot z}}+\cdots+w^{l_{s} h_{s \cdot z}}\right)^{z}=w^{l_{1 \cdot z-1} h_{1}}+\cdots+w^{l_{s \cdot z}-1} h_{s},
$$

whereas $v^{x}=w^{l_{1 \cdot z^{-1}}}+\cdots+w^{l_{s \cdot z^{-1}}}$; setting $u=l_{i \cdot z^{-1}} \in H$, we deduce that $w^{u h_{i}}=w^{u}$, a contradiction that proves our claim.

As regards (b), take $\left(\Sigma_{1}, \Sigma_{2}, \ldots, \Sigma_{t+1}\right)$ in a $k$-deranged orbit for the action of $K$ on $\mathcal{P}_{t}(\Sigma)$, and choose $r_{1}, r_{2}, \ldots, r_{t+1} \in W$ in pairwise distinct $H$-orbits; considering the element $v=w_{1}+\cdots+w_{s} \in W^{\oplus s}$ such that $w_{i}=r_{j}$ if $i$ lies in $\Sigma_{j}$, we claim that $v$ lies in a $g$-deranged orbit for the action of $G$ on $V$. In fact, let $x \in G$ be such that $v^{x g x^{-1}}=v$. Writing $x=\left(l_{1}, \ldots, l_{s}\right) z$ as in part $(\mathrm{a})$, we get $g^{x^{-1}}=\left(y_{1}, y_{2}, \ldots, y_{s}\right) k^{z^{-1}}$ for suitable $y_{i} \in H$, whence, setting $b=k^{z^{-1}}$,

$$
w_{1 \cdot b^{-1}}^{y_{1 \cdot b-1}}+\cdots+w_{s \cdot b^{-1}}^{y_{s \cdot b-1}}=w_{1}+\cdots+w_{s} .
$$

It is not hard to deduce that, in this situation, $b=k^{z^{-1}}$ is forced to stabilize $\Sigma_{j}$ for every $j \in\{1, \ldots, t+1\}$, thus contradicting the choice of $\left(\Sigma_{1}, \Sigma_{2}, \ldots, \Sigma_{t+1}\right)$ as an element of $\mathcal{P}_{t}(\Sigma)$ lying in a $k$-deranged orbit, and the proof is complete.

Finally, we consider irreducible linear actions of solvable groups.

Theorem 2.6. Let $G$ be a solvable group, $p$ a prime number greater than 3 , and $V$ a faithful irreducible $G$-module over a prime field. Let $g$ be a p-regular element of $G$ such that there do not exist $g$-deranged orbits in $V$. Then $g$ lies in $\mathbf{O}_{p^{\prime} p p^{\prime}}(G)$, unless $p \in\{5,7\}$ and the order of $g$ is divisible by 2 or 3 .

Proof. We can clearly assume $g \neq 1$. Since Theorem 2.2 settles the primitive case, we can assume that the action of $G$ on $V$ is imprimitive, and we will use 
Notation 2.4. So, let us write $g=\left(h_{1}, h_{2}, \ldots, h_{s}\right) k$. If $k=1$, then Lemma 2.5(a) yields that, for every $i \in\{1, \ldots, s\}$, there does not exist any $h_{i}$-deranged orbit for the action of $H$ on $W$; as $g$ is nontrivial, there certainly exists $i \in\{1, \ldots, s\}$ such that $h_{i}$ is nontrivial, therefore Theorem 2.2 yields $H=\mathbf{O}_{p^{\prime} p p^{\prime}}(H)$ and the desired conclusion easily follows. In view of this, we will henceforth assume $k \neq 1$.

Observe that, by Theorem 1.4(b), there exists a $k$-deranged orbit for the action of $K$ on $\mathcal{P}_{3}(\Sigma)$; as a consequence of this fact together with Lemma 2.5(b), we get that $W$ is partitioned in at most three orbits under the action of $H$, hence Lemma 2.3 yields $H=\mathbf{O}_{p^{\prime} p p^{\prime}}(H)$. Now we look at the action of $K$ on $\mathcal{P}(\Sigma)$ : the existence of $k$-deranged orbits for this action would imply, again by Lemma 2.5(b), the existence of a $g$-deranged orbit for the action of $G$ on $V$. As this is not the case by our assumptions, in view of Theorem 1.4(d) we can hence conclude that $k$ is a $\{2,3\}$-element of $K$ (so the order of $g$ is divisible by 2 or 3 ) and that $k$ lies either in $\mathbf{O}_{\{2,3,5\}}(G)$ or in $\mathbf{O}_{\{2,3,7\}}(G)$. It is then clear that $g$ lies in $\mathbf{O}_{p^{\prime} p p^{\prime}}(G)$ unless $p$ is either 5 or 7 , and the proof is complete.

\section{Proof of Theorem A}

We are ready to prove Theorem A, that was stated in the Introduction. The argument is essentially the same as in Theorem A of [3], but of course here we will be using Theorem 2.6, instead of Theorem $\mathrm{C}$ of that paper.

Proof of Theorem A. Assume either $p>7$, or $p>3$ with the order of $g$ coprime to 6 , and observe that the hypotheses of the theorem are inherited by the element $g \boldsymbol{\Phi}(G)$ of the factor group $G / \boldsymbol{\Phi}(G)$ (where $\boldsymbol{\Phi}(G)$ denotes the Frattini subgroup of $G)$. In view of this fact, it will be enough to prove Theorem $\mathrm{A}$ in the case when $\boldsymbol{\Phi}(G)=1$; this extra assumption ensures that $F:=\mathbf{F}(G)$ is a completely reducible $G$-module (possibly in "mixed characteristic").

Let $V$ be a minimal normal subgroup of $G$. Then $\widehat{V}=\operatorname{Irr}(V)=\operatorname{IBr}_{p}(V)$ (recall that $p$ does not divide $|V|)$ is a faithful irreducible $G / \mathbf{C}_{G}(V)$ module. Take $\mu \in \widehat{V}$ and let $\phi \in \operatorname{IBr}_{p}(G)$ lying over $\mu$. By Clifford correspondence (see for instance [9, $(8.9)]), \phi$ is induced by an irreducible Brauer character of $I_{G}(\mu)$, and therefore it vanishes on every $p$-regular element of $G$ not belonging to the set $S=\bigcup_{x \in G} I_{G}\left(\mu^{x}\right)$. Since the Brauer character $\phi$ does not vanish on $g$, we get $g \in S$, and hence we conclude that there are no $g \mathbf{C}_{G}(V)$-deranged orbits for the action of $G / \mathbf{C}_{G}(V)$ on $\widehat{V}$. We can now apply Theorem 2.6, getting that $g \mathbf{C}_{G}(V)$ lies in $\mathbf{O}_{p^{\prime} p p^{\prime}}\left(G / \mathbf{C}_{G}(V)\right)$.

Writing $F=V_{1} \times \cdots \times V_{n}$ where the $V_{i}$ are minimal normal subgroups of $G$, and observing that $F=\bigcap_{i=1}^{n} \mathbf{C}_{G}\left(V_{i}\right)$, the result now follows because $G / F$ can be regarded as a subgroup of $G / \mathbf{C}_{G}\left(V_{1}\right) \times \cdots \times G / \mathbf{C}_{G}\left(V_{n}\right)$, hence $g F$ lies in $\mathbf{O}_{p^{\prime} p p^{\prime}}(G / F)$, and finally $g$ lies in $\mathbf{O}_{p^{\prime} p p^{\prime}}(G)$ because $p$ does not divide the order of $F$.

\section{EXAmples}

In order to round out our understanding of the subject, we devote the last section to some examples. The first of them shows that Theorem A is somewhat "optimal".

Example 4.1. Let $G=A \Gamma\left(2^{7}\right)$ be the affine semilinear group on $\operatorname{GF}\left(2^{7}\right)$. Then, for $p=127, \mathbf{O}_{p}(G)=1$ and the $p$-Brauer character table of $G$ contains no zeros. In particular, every element of order 7 of $G$ is a $p$-nonvanishing element of $G$ not belonging to $\mathbf{O}_{p^{\prime} p}(G)$. 
Next, we see that Theorem 2.6 is false in general when $p \in\{5,7\}$ and the order of $g$ is not coprime with 6 .

Example 4.2. Let $H=\Gamma\left(2^{4}\right)$, acting on its natural module $W=\mathrm{GF}\left(2^{4}\right)^{+}$, and let $K$ be a subgroup of $S_{5}$ with $K \simeq D_{10}$. Consider $G=H$ < $K$ acting on the irreducible module $V=\oplus_{i=1}^{5} W_{i}$, where $\left|W_{i}\right|=|W|=2^{4}$ for $i \in \Sigma=\{1, \ldots, 5\}$. Let $g$ be an involution of $K$, let $v=\sum_{i=1}^{5} w_{i} \in V$ (where $w_{i} \in W_{i}$ ), and set $I_{v}=\left\{i \mid w_{i} \neq 0\right\} \in \mathcal{P}(\Sigma)$. Now, as $g$ is in the stabilizer of a $K$-conjugate of $I_{v}$, taking into account that $H$ is transitive on $W \backslash\{0\}$, it is not hard to see that $g$ centralizes a $G$-conjugate of $v$. Thus, there are no $g$-deranged orbits under the action of $G$ on $V$ and, for $p=5, g$ is a $p$-regular element not lying in $\mathbf{O}_{p^{\prime} p p^{\prime}}(G)$.

A similar example can be made for $p=7$, with the element $g$ having order 3 . Let $H=\Gamma\left(2^{3}\right)$ act on its natural module $W$ of order $2^{3}$, and let $K=A \Gamma\left(2^{3}\right)$ act naturally on 8 elements. Consider the corresponding action of $G=H$ 々 $K$ on $V$ with $|V|=2^{24}$. If $g$ is an element of order 3 of $K$, then there are no $g$-deranged orbits under the action of $G$ on $V$ and, for $p=7, g$ is a $p$-regular element not lying in $\mathbf{O}_{p^{\prime} p p^{\prime}}(G)$.

\section{ACKNOWLEDGEMENTS}

The second author wishes to express his deepest gratitude to Laci Kovács, an invaluable teacher and an inspiring mentor, well beyond Mathematics. He also thanks the Editors for the invitation to submit a paper for the special issue dedicated to Laci's memory.

\section{REFERENCES}

[1] S. Dolfi, Large orbits in coprime actions of solvable groups, Trans. Amer. Math. Soc. 360 (2008) 135-152.

[2] S. Dolfi, Orbits of permutation groups on the power set, Arch. Math. 75 (2000) 321-327.

[3] S. Dolfi, E. Pacifici, Zeros of Brauer characters and linear actions of finite groups, J. Algebra 340 (2011) 104-113.

[4] S. Dolfi, G. Navarro, E. Pacifici, L. Sanus and P.H. Tiep, Non-vanishing elements of finite groups, J. Algebra 323 (2010) 540-545.

[5] D. Foulser, Solvable primitive permutation groups of low rank, Trans. Amer. Math. Soc. 143 (1969) 1-54.

[6] I. M. Isaacs, Character Theory of Finite Groups, Dover, New York, 1976.

[7] I.M. Isaacs, G. Navarro, T.R. Wolf, Finite group elements where no irreducible character vanishes, J. Algebra 222 (1999) 413-423.

[8] O. Manz, T.R. Wolf, Representations of solvable groups, Cambridge University Press, Cambridge, 1993.

[9] G. Navarro, Characters and blocks of finite groups, Cambridge University Press, Cambridge, 1998.

[10] A. Seress, The minimal base size of primitive solvable permutation groups, J. London Math. Soc. 53 (1996) 243-255.

[11] Y. Yang, Regular orbits of finite primitive solvable groups, II, J. Algebra 341 (2011) 23-34.

Silvio Dolfi, Dipartimento di Matematica U. Dini,

Università degli Studi di Firenze, viale Morgagni 67/A, 50134 Firenze, Italy.

E-mail address: dolfi@math.unifi.it

Emanuele Pacifici, Dipartimento di Matematica F. Enriques,

Università degli Studi di Milano, via Saldini 50, 20133 Milano, Italy.

E-mail address: emanuele.pacifici@unimi.it

Lucia Sanus, Departament d’Àlgebra, Facultat de Matemàtiques,

Universitat de València, 46100 Burjassot, València, Spain.

E-mail address: 1sanus@uv.es 